\title{
Lymphovascular malformations, imaging spectrum on USG and CT (with emphasis on intralesional haemorrhage) - A descriptive study
}

\author{
Maravi $\mathbf{P}^{1}$, Soni $\mathbf{N}^{2}$, Kaushal $\mathbf{L}^{3}$ \\ ${ }^{1}$ Dr Poornima Maravi, Assistant Professor, ${ }^{2}$ Dr Neelam Soni, Junior Resident, ${ }^{3}$ Dr Lovely Kaushal Professor and Head. \\ All are affiliated with Department of Radiology and Imaging, GMC, Bhopal, Madhya Pradesh, India.
}

Address for Correspondence: Dr Poornima Maravi, Email: maravipoornima@ gmail.com

\begin{abstract}
Introduction- Lymphovascular/lymphatic malformations usually affect infants or children. Most common sites are the head and neck. When, superficial, they present with growing swelling in affected part. Their preoperative evaluation is necessary for better postoperative outcome and to reduce the chances of recurrence. Ultrasound is the useful modality to depict the exact location and character of lesion. Colour Doppler demonstrates vascular nature of lesions. CECT providing three dimensional views of lesion as well as insinuation/extension in adjacent structures, ensuring complete surgical removal of lesion and better postoperative outcome. Method -We studied imaging spectrum of LVMs (lymphovascular malformations) in USG and colour Doppler in 40 patients at Gandhi medical college, Bhopal.29 patients have further undergone CECT for better depiction of lesion. We noted imaging spectrum of lesions on USG and CECT. Results-Most patients presenting with growing/congenital swelling were infants. Most common finding in USG was complex multisptated cystic lesion showing intraseptal vascularity on Doppler. CECT demonstrated multiseptated cystic lesion with peripheral and septal enhancement. Most of them were fluid density lesion and showing insinuation in adjacent structure. 18 patients were confirmed on FNAC and 22 patients were confirmed on surgery. Conclusion-USG is best mean to describe the character and location of lesion, also widely available, easy to use and suitable for infants and children (no sedation required). Many cases require further imaging with CECT, for further characterization of lesion and to evaluate their extension/insinuation. Surgical removal is final treatment keeping the incidences of recurrences low.
\end{abstract}

Keywords: Lymphovascular /Lymphatic Malformations, CDUS=Colour Doppler Ultrasound, CECT=Contrast Enhanced Computed Tomography,

\section{Introduction}

Lymphovascular malformations are rare group of benign proliferative developmental anomalies of lymphatic system (1-3)They are caused by failure of developing lymphatic tissue to establish a normal communication with remainder of the lymphatic system. These abnormal lymphatic channels then dilate to form a unilocular or multilocular cystic mass (4).

The lymphatic system arises from 6 primitive sacs that develop at 6 th week of intrauterine life. First pair is jugular sac, second pair is cysterna chyli located at retroperitoneal tissues, and third pair is posterior lymph sacs develop at inguinal region. Lymphatic malformations can affect any area of the body except

Manuscript received: $24^{\text {th }}$ Oct 2015

Reviewed: $10^{\text {th }}$ Nov 2015

Author Corrected: $20^{\text {th }}$ Nov 2015

Accepted for Publication: $30^{\text {st }}$ Nov 2015 the brain, most common sites are the head and neck, and less common locations include the mesentery, retroperitoneum, abdominal viscera, lung and mediastinum. When superficial, they present with growing swelling in head /neck, trunk or, extremities. Patient is usually an infant or child, sometimes an adolescent or adult. When they get complicated, (secondary infection/ intralesional haemorrhage), they are detected by pain, tenderness or discolouration over the swelling. Lymphatic malformation represents less than $5 \%$ of all congenital neck masses in children and even smaller amount in adults (5).

Plain X-ray is of limited use, detecting soft tissue swelling over the part or sometimes punctate calcifications due to the phleboliths. Ultrasound is the useful modality to depict the exact location and character of lesion.USG imaging usually show well 
defined, thin walled, multilocular or rarely unilocular, cystic masses that are anechoic or sometimes contain echogenic debris (secondary infection/haemorrhage).Colour Doppler may demonstrate peripheral/ intraseptal vascularity. The presence of haemorrhage or infection in the lesion gives a more complex appearance with multiple thin septations, coarse/fine echoes, echogenic debris and thick appreciable wall (6).Contrast enhanced computed tomography is investigation of choice which shows peripheral and septal enhancement. Insinuation in adjacent structures is well depicted due to its three dimensional ability. Compression of adjacent structures (nerve/artery) or pressure erosion of underlying bone can be seen in rare cases. The fluid component is typically homogeneous with fluid attenuation values (1$10 \mathrm{HU})$, may be more (10-20 HU), when complicated by secondary infection or haemorrhage. Sometimes negative attenuation values occur in the presence of chyle or air (due to interventions) (7). Most cases require treatment, due to growing swelling causing facial disfigurement, compression of adjacent structure or due to the complications (haemorrhage/infection) (2, 6). Treatment of choice is direct surgical removal. However recurrences are common due to insinuation of lesion in adjacent structures and thus leading to complete removal difficult. Thus imaging is important, demonstrating accurate anatomic localization, character and definition of the lesion for pre operative planning.

\section{Method}

The study was conducted in Gandhi Medical College and Hamidia Hospital, Bhopal for 6 months. Our series consists of 40 patients, presenting with growing/congenital swelling in different parts of the body. The age of the patients ranged between 2 days to 27 years .Most patients were referred from surgical and paediatric department. A Philips HD7 colour Doppler scanner with 7 to $12 \mathrm{MHz}$ linear transducers was used for examination. Few infants were examined sedated, which was helpful to make them calm and to obtain high quality spectral waveform from lesion. CECT was performed in WIPRO GE machine.Patients presenting with superficial swellings with characteristic findings of lymphovascular malformations on USG were included. Patients were followed up for CECT findings, further confirmation of diagnosis by FNAC/Surgery and for final treatment obtained.

\section{Results}

In this descriptive, hospital based study, 12 patients were infants; 10 patients were children up to fourteen years of age. 8 patients were from age group 15-19. Rest were between 20-30 yrs. 35 patients were male and 5 patients were female. Most patients were referred from surgical department complaining growing swelling over the affected part. 7 patients out of 12 infants presented with congenital swelling.6 patients presented with enlargement and discolouration of swelling. 7 patients presented with postoperative recurrence of swelling. Infants and children were directly referred for the ultrasound.

13 swellings were located in the neck (diffusely involving the neck in 8 patients, posterior triangle in 3 patients and anterior triangle in 2 patients), 5 in axillary region, 4 swellings were located in one of the cheek ( 3 were on right cheek and 1 were on left cheek), 3 in submandibular region, 3 in supraclavicular region, 4 were located in anterior abdominal wall (in which three were the case of postoperative recurrence after 2-3 yrs). 2 were located over the chest, 1 was over the saccrococcygeal region, 1 was located on left forearm and hand extending up to the $3^{\text {rd }}$ and $4^{\text {th }}$ digits dorsally, 1 was located in left arm ,1 was small and located over the ring finger of left hand, 1 over the lateral melleolus of right leg. 29 patients have further undergone computed tomography for evaluating the extension of lesion. The diagnosis of LVMs was confirmed by surgery in 22 patients and FNAC in 18 patients.

One, female child patient with a large diffuse swelling over right forearm and hand with history of postoperative recurrence after three years of surgical removal showed increased density and moulding of underlying ulna on $\mathrm{x}$ ray left forearm.

All patients were under gone ultrasound on the same day of referral. Ultrasound of local part performed using 7-12 $\mathrm{MHz}$ linear transducer which demonstrated the thin walled, multiloculated, multi septated, (sometimes unilocular) cystic swelling, many of them demonstrating intraseptal vasculrity on colour Doppler assessment.

Lymphatic malformations are most commonly located in the head and neck. The main division in classifying lymphatic malformations is whether they contain macrocysts $(>2 \mathrm{~cm})$, microcysts $(<2 \mathrm{~cm})$, or both. Macrocystic lesions are more easily treated and carry a better prognosis than its microcystic counterpart [8]. 
One 15 year old male child presented with a large, recurrent, nodular lump overhanging from lower abdominal wall and grossly distended abdomen. USG abdomen revealed USG and CT showed characteristic imaging findings of lymphovascular malformations. Small umbilical hernia was also noted. Hypoechoic, homogenous thrombus also seen in the root of IVC. Gross ascites and minimal pericardial effusion were also seen. These changes may be due to massive venous
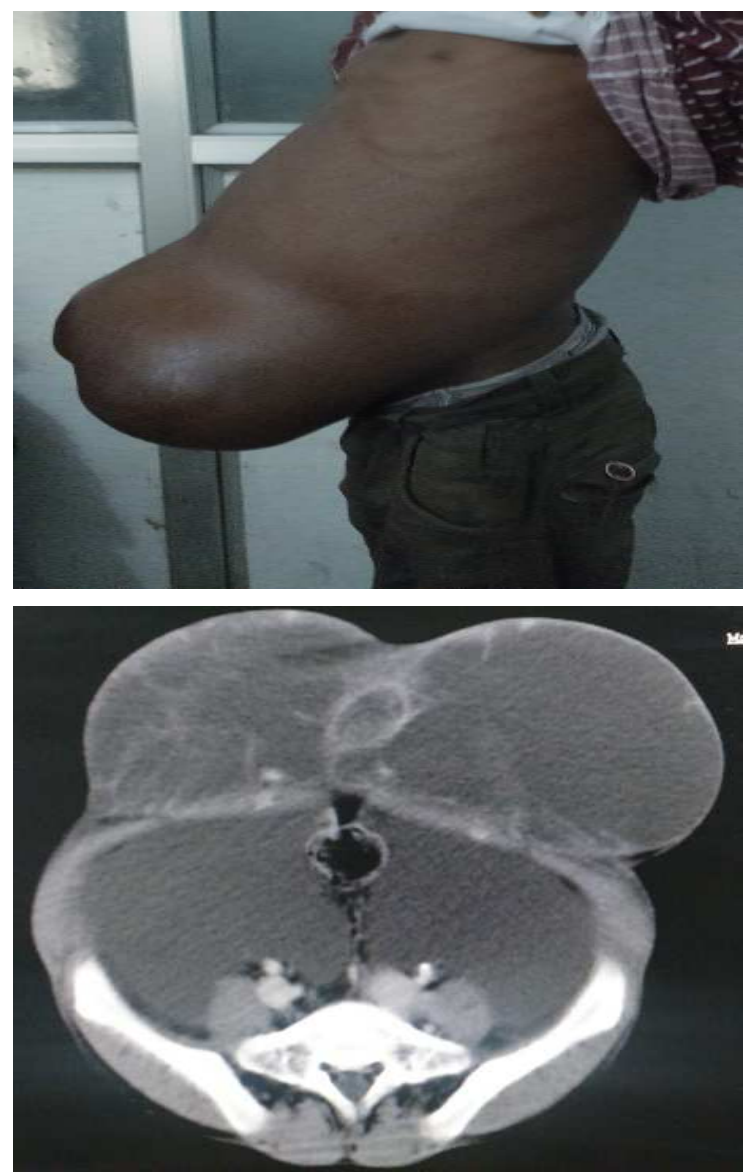

return leading to right sided cardiac overload and subsequent right sided cardiac failure. Abdominal lymphangiomas are uncommon benign tumours, usually presenting in early childhood as a palpable abdominal mass. Their Sonographic and CT appearance has been well described. Rarely, they may be large enough to be confused with ascites on physical exam. Limited literature is available on abdominal wall lymphangioma [9].
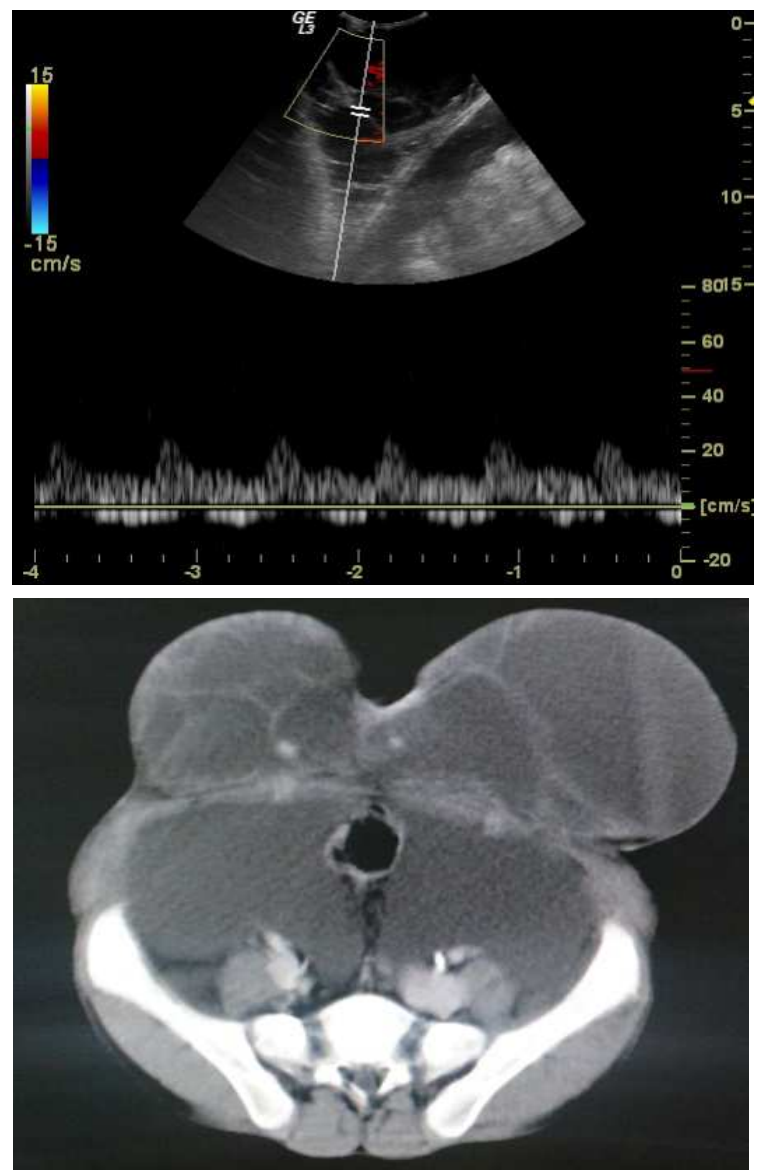

Figure 1: showing large nodular swelling hanging from abdominal wall. USG showing complex multiseptated cystic swelling with prominent intraseptal arterial flow \& CT axial contrast images multiloculated lesion with peripheral and septal enhancement.

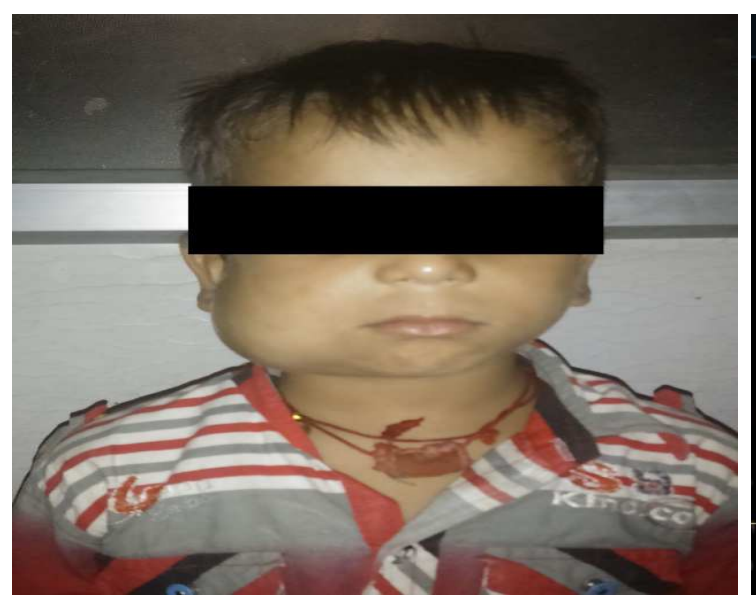

International Journal of Medical Research and Review

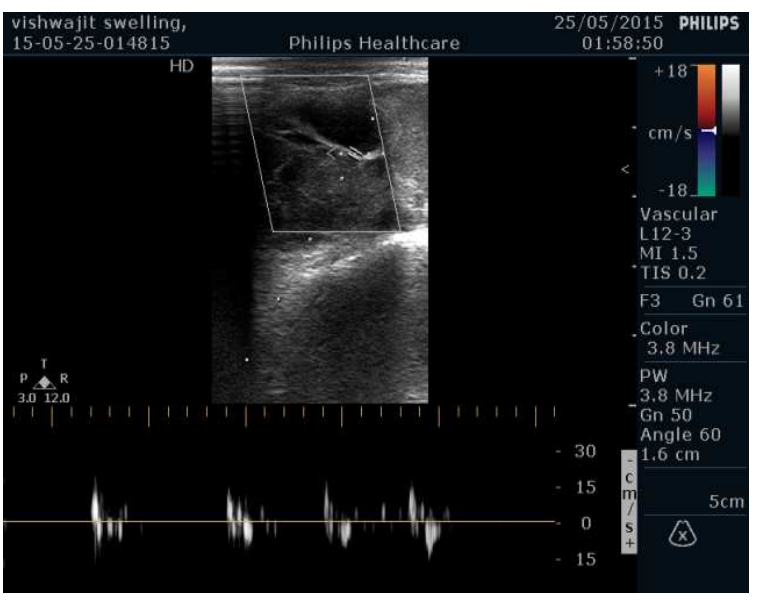

Available online at: $\underline{\text { www.ijmrr.in }} 1259$ | P a g e 
Figure 2: cystic swelling over right cheek \& USG images showing multiseptated,cystic lesion showing low velocity intraseptal arterial flow and echogenic thrombus.

6 patients aged 6-10 yrs, presented with enlargement and discoloration of swelling. One 10 year old, male child showed compressible, tender swelling on right cheek, Ultrasound showed features suggestive of LVMs with prominent arterial flow on Doppler assessment. The lesion demonstrated multiple, floating, fine echoes with a large echogenic fresh thrombus (fresh haemorrhage within the lesion). Histopathology, confirmed the lesion to be hemangiolymphoma.Patient followed up two weeks later showed reduction of size of lesion with multiple reticular septations suggesting resolving bleed.

\section{Discussion}

In the literature nearly all reported cases of the lymphatic malformation are diffuse cystic hygroma in the posterior triangle of the neck in infants or newborns. History of trauma may be associated with some lesions (10, 11-15), but in present series, no significant history of trauma was found. They are most commonly found in infants, followed by children and adolescents. Since these are primarily fluid filled and are usually superficial in location, they are easily examined with high-resolution ultrasound. USG is primary, inexpensive, nonionising, noninvasive imaging modality (10).

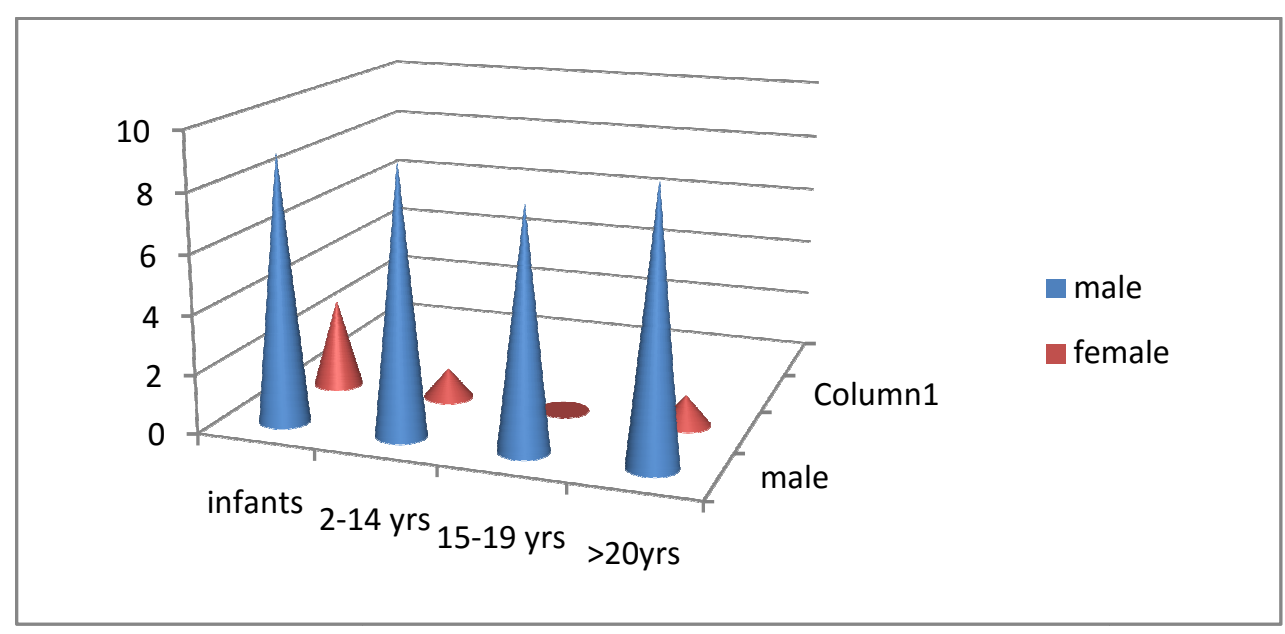

Age and sex distribution:

-Most patients were infants, with clear male preponderance.

The etiology of lymphangiomas is probably a congenital abnormality of the lymphatic system, causing sequestrations of lymphatic tissue during embryologic development (16). This theory would explain why lymphangiomas occur primarily in children. However, it is suggested that abdominal trauma, lymphatic obstruction, inflammatory process, surgery, or radiation therapy may lead to the secondary formation of such a tumor $(17,18)$. Traditionally, lymphangiomas are classified as simple, cavernous, or cystic. The simple type is usually situated superficially in the skin and composed of small thin-walled lymphatic vessels. The cavernous type is composed of dilated lymphatic vessels and lymphoid stroma, and has a connection with spaces of various normal adjacent lymphatics. Lastly, the cystic type consists of lymphatic spaces of various sizes that contains fascicles of smooth muscle and collagen bundles, but has no connection with adjacent normal lymphatics. However, cystic lymphangioma is not always clearly differentiated from cavernous type because the cystic type may also contain cavernous areas (19).

Head and neck is a common site of involvement (21).About $75 \%$ of LVMs occur in the neck, and $20 \%$ occur in the axillary region, which was consistent with our study. In our study, most patients were infants (33\%) with history of congenital or slow growing swelling over neck. In most cases, swelling was diffuse involving the neck, restricting the neck movements (6). 


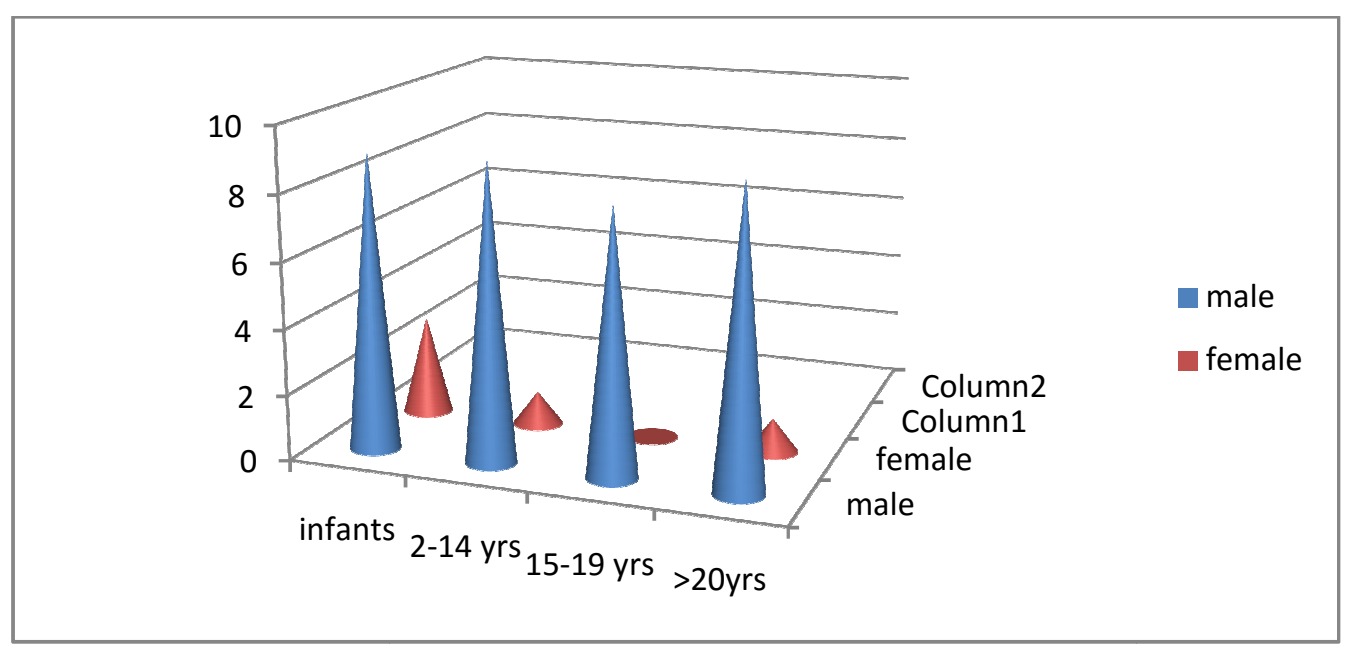

-Most patients were presented with congenital swelling neck (33\%), followed by axillary swellings (13\%).

Most infants were 1-11 months old. Other locations include axillary, chest, submandibular, supraclavicular, saccrococcygeal region, extremities and digits. However, they may also found in mediastinum, abdominal viscera, retroperitoneum, bones, and scrotum. In our study all lesion were superficial and easily accessible to examination. Approximately $50-60 \%$ 0ccur at birth and about $80-90 \%$ occur before the completion of second year (2). A few cases have been reported in the adult population but incidence is very low compared to that of children (22-24). In our study earliest age of presentation was 2 days old male infant presenting with congenital swelling in neck.

Though no sex predilection has been described in previous studies, our study showed male preponderance. Correlation of the sonogram with the pathologic specimen demonstrated that the echogenic component corresponded to a cluster of abnormal lymphatic channels, too small to be resolved with ultrasound. Large lesions had ill-defined boundaries, with cystic components dissecting between normal tissue planes. Sonographically, one can usually differentiate these tumors from other cervical masses, especially soft-tissue hemangiomas. Sonography is also helpful in determining the extent of the lesion before surgery and in assessing postoperative complications and recurrences (16).

On Sonography, LVMs appear as unilocular or more commonly multilocular cystic masses that are anechoic or contain echogenic debris (sometimes). They are usually slow growing masses but sudden enlargement can occur owing to internal haemorrhage, inflammation or even respiratory tract infection or trauma .In our study, six patients presented with enlargement and discolouration of swelling, of which, two demonstrated fresh intralesional bleed with echogenic thrombus

These Sonographic appearances of LVMs were encountered: (a)macrocystic with thin and thick septae; (b) macrocystic with thin septae and dense, floating echoes (consistent with fresh haemorrhage/infection) and (c) mixed microcystic and macrocystic, with thin septations (d) microcystic ,ill defined mass with thick septations and adjacent scarring (Mostly consisting of post operative cases).Most common finding on USG was, a typical multiloculated, multisepatated cystic swelling with thin internal septations, Which may or may not show vascularity.

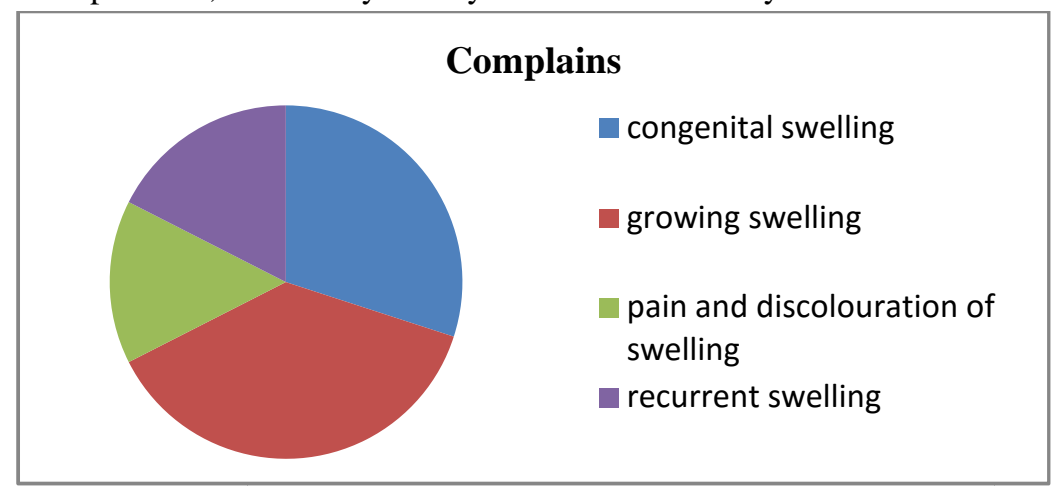




\section{-Most patients presented with growing swelling. \\ -Most infants presented with congenital swelling. \\ $-18 \%$ presents with post op recurrences of swelling.}

Basically that can be divided into capillary, cavernous, cystic hygromas and mixed variety as per endothelial characteristics which determine flow within the lesion (25). Jackson divided vascular malformations into two types as per flow characteristics: high flow and low flow; management of two differs exquisitely. Lymphatic malformations were classified as low flow lesions (26).Total 75\% patient's demonstrated internal vascularity, of which $56.66 \%$ showed venous flow, categorizing the most of lesions in slow flow LVMs. Rest showed either arterial $(26.66 \%)$ or mixed $(16.66 \%)$ vascularity or no vascularity $(25 \%)$. Velocity ranged from 6-20 cm/sec.

In our study 29 patients were undergone CT for further evaluation of lesion. Most characteristic CT finding was complex, cystic lesion with peripheral and septal enhancement. The lesion was mostly of fluid density ranged from 4-13 HU. However, density may be more if, haemorrhage/infection is there (18-25 in our cases of intralesional haemorrhage) or may be negative in presence of air or chyle. Most lesion were faintly enhancing, depicting slow flow lymphatic venous malformations.

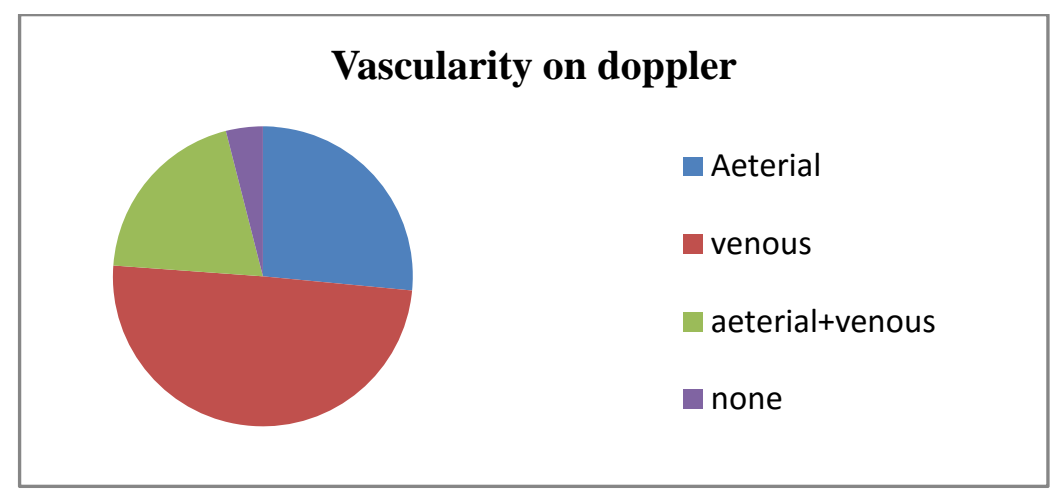

-In most cases venous flow was predominant, categorising them in slow flow lymphovascular malformation.

Sheth S et al (20) correlated Sonographic findings with pathologic specimen and demonstrated that the echogenic component corresponded to a cluster of abnormal lymphatic channels, too small to be resolved with ultrasound. They also reported calcified focus in one of their case, which on histology was proved to be due to calcified thrombus. However, in our study no calcified foci were detected. Sometimes LVMs cannot be confidently diagnosed preoperatively, when complicated by haemorrhage or infection. Ultrasound is very helpful in determining the extent of cystic hygromas before surgery; However cross sectional study is needed to evaluate the proper extent of lesion and assessing postoperative complications and recurrences. The differential diagnosis of a predominantly cystic neck mass included branchial cyst, thyroglossal duct cyst, abscess, resolving hematoma, lymph node, teratoma, laryngocoele, orpharyngocoele.

Diagnosis can be confirmed by histology and surgery. In our study $45 \%$ patients were confirmed on FNAC and 55\% were confirmed on surgery and removal of mass.

The treatment of choice is surgical excision of localized LVMs, but surgeons often worry about the infiltrative nature of some lesions and the difficulty in achieving complete resection. The resection has traditionally been through open surgery; however laparoscopic resection has been reported. Treatment of lymphatic malformation differs that from other vascular malformations. Different treatment modalities have been suggested by various authors including, aspiration, radiation, sclerosants, and surgery. Aspiration may be useful for emergency decompression but is not a definitive treatment.

Recurrence rate is found to be $10-38 \%$ and is directly related to the excisional surgery (10). Recurrences may be found in late stages (in our study 27 yrs old male presented with recurrent swelling).In our series seven patients presented with postop recurrence of swelling. Near all of them exhibiting microcystic variety on imaging. The mean duration of recurrence was 2 yrs. 


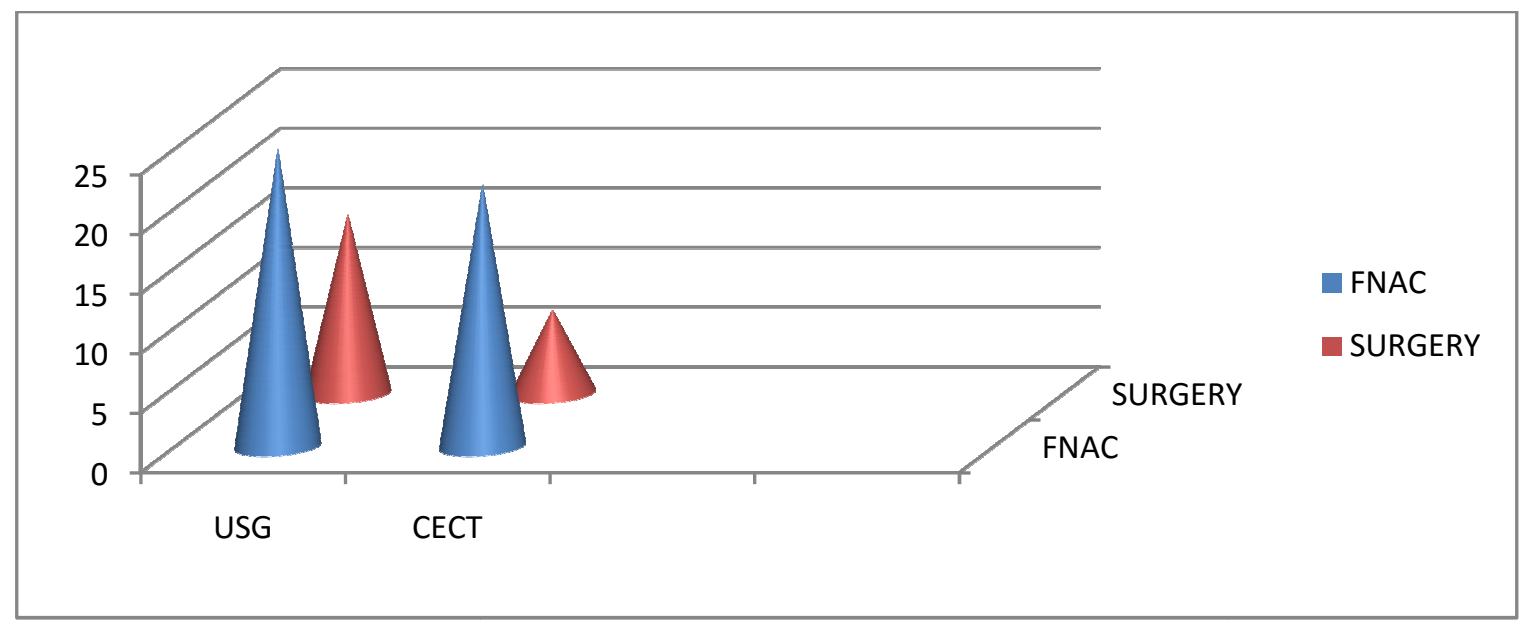

\section{Conclusion}

All LVMs are more or less similar on imaging, until complicated by haemorrhage/infection. All LVMs should be evaluated preoperatively by USG and cross sectional imaging to evaluate the nature and extent of lesion.USG is excellent primary modality to show its cystic, multiloculated nature while giving the insight about the vascularity (arterial, venous, mixed). Imaging plays an important role in accurate anatomic localization, characterization and extension of the lesions for preoperative planning because LVMs have an insinuating nature that makes complete surgical excision difficult in many cases .Intra-lesional haemorrhages were common than secondary infections and were easily picked up by imaging. Recurrences were also common after surgical removal and their treatment needs multimodality approach.

\section{Funding: None}

Competing interests: None declared

Ethical clearance: The study was approved by the institutional human ethical committee

\section{References}

1. Smith R, Burke DK, et al.OK-32 therapy for lymphangiomas: Arch Otolaryngol Head Neck Surg.1996; References 122:1195-1199.

2. Mandel L. Parotid area lymphangiomas in an adult: case report. J Oral Maxillofac Surg.2004; 62(10):13201323. doi: 10.1016/j.joms.2003.12.040.

3. Bailey CM. Cystic hygroma. Lancet. 1990; 335:511512. doi: 10.1016/0140- 6736(90)92683-9.
4. Iyer R, Eftekhari F, Varma D, Jaffe N. Cystic retroperitoneal lymphangioma: $\mathrm{CT}$, ultrasound and $\mathrm{MR}$ findings.Pediatr Radiol. 1993; 23(4):305-6.

5. Machado LE, Osborne NG, Bonilla-Musoles F. Three-dimensional sonographic diagnosis of lymphangiomas. J Ultrasound Med. 2004; 23(6):877881.

6. Kraus R, Han BK, Babcock DS, Oestreich AE. Sonography of neck masses in children.AJR 1986, 146:609-613.

7.Davidson AJ, Hartman DS. Lymphangioma of the retroperitoneum:CT and Sonographic characteristics Radiology. 1990 May; 175(2):507-10

8. Buckmiller LM, Richter GT, Suen JY. Diagnosis and management of hemangiomas and vascular malformations of the head and neck. Oral Dis. 2010;16:405-418.

9. Lugo-Olivier $\mathrm{CH}$, Taylor GA. CT differentiation of large abdominal lymphangioma from ascites. PediatrRadiol 1993; 23:129-130

10. Mandel L. Parotid area lymphangiomas in an adult: case report. J Oral Maxillofac Surg.2004; 62(10):1320 1323. doi: 10.1016/j.joms.2003.12.040.

11. Toranzo JM, Guerrero F, et al. A congenital neck mass. Oral Surg Oral Med Oral Pathol.1996;82(4):363364. 
12. Osborne TE, Alex Haller J, et al. Submandibular cystic hygroma resembling a plunging ranula in a neonate-review and report of a case. Oral Surg Oral Med Oral Pathol.1991;71(1):16-20. Doi: 10.1016/0030-4220(91)90513-C.

13.Saijo M, Munro IR, Mancer K. Lymphangioma-a long term follow-up study. Plast Reconstr Surg.1975; 56:642-651. doi: 10.1097/00006534-19751100000006.

14. Fraser SE, Campbell B, et al. Pathologic quiz 2. Arch Otolaryngol Head Neck Surg. 1996; 122:1200.

15. Hudson JW, Meszaros EJ. Submucosal lymphangiomas of the maxillary sinus. $\mathbf{J}$ Oral and Maxillofacial Surgery. 2003; 61: 390-392.

16. Roisman I, Manny J, Fields S, Shiloni E. Intraabdominal lymphangioma. Br J Surg. 1989; 76:485489.

17. Hardin WJ, Hardy JD. Mesenteric cysts. Is J Surg. 1970; 119:640-645. [PubMed]

18. Daniel S, Lazarevic B, Attia A. Lymphangioma of the mesentery of the jejunum: report of a case and a brief review of the literature. Am J Gastroenterol. 1983; 78:726-729.

19..Rieker RJ, Quentmeier A, Weiss C, Kretzschmar U, Amann K, Mechtersheimer G, Bläker H, Herwart OF. Cystic lymphangioma of the small-bowel mesentery: case report and a review of the literature. Pathol Oncol Res. 2000;6:146-148.
20. Sheth S, Nussbaum AR, Hutchins GM, Sanders RC. Cystic hygromas in children. Sonographic pathologic correlation, Radiology 1987, 162:821-824.

21. Serres MD, Sie KCY, et al. Lymphatic malformations of the head and neck: proposal for staging. Arch Otolaryngol Head Neck Surg. 1995; 121(5):577-582.

22.Goodman P, Yeung CS, Batsakis JG. Retropharyngeal lymphangioma presenting in an adult.Otolaryngol Head Neck Surg. 1990; 103(3):476479.

23.Schefter RP, Olsen KD, Gaffey TA. Cervical lymphangioma in the adult. Otolaryngol Head Neck Surg. 1985; 93(1):65-69.

24.Muñoz Herrera A, Pérez Plasencia D, Gómez Benito M, Santa Cruz Ruiz S, Flores Corral T, Aguirre García F. Cervical lymphangioma in adults- Description of 2 cases. Ann Otolaryngol Chir Cervicofac.1998; 115(5):299-302.

25. Mulliken JB, Glowacki J. Hemangiomas and vascular malformations in infants and children: a new classification based on endothelial characteristics. Plast Reconstr Surg. 1982; 69:412-422. doi: 10.1097/00006534-198203000-00002.

26. Jackson IT, Carreno R, et al. Haemangioma, lymphatic malformations and lymphovenous malformations: classification and methods of treatment. Plast Reconstr Surg. 1993; 91(7):1216-1230. doi: 10.1097/00006534-199306000-00006.

\section{How to cite this article?}

Maravi P, Soni N, Kaushal L. Lymphovascular malformations, imaging spectrum on USG and CT (with emphasis on intralesional haemorrhage) - A descriptive study. Int J Med Res Rev 2015;3(10):1257-1264. doi: 10.17511/ijmrr.2015.i10.228. 